\title{
Depressor and Tachypneic Responses to Chemical Stimulation of the Ventral Respiratory Group Are Reduced by Ablation of Neurokinin-1 Receptor-Expressing Neurons
}

\author{
Hong Wang, Teresa P. Germanson, and Patrice G. Guyenet \\ Department of Pharmacology, University of Virginia, Charlottesville, Virginia 22908
}

\begin{abstract}
Our goal was to investigate whether the neurokinin-1 receptor (NK1R)-expressing cells of the rostral ventrolateral medulla (RVLM) regulate respiration and arterial pressure (AP). We examined the consequences of their ablation on the cardiorespiratory responses [phrenic nerve discharge (PND) and AP] caused by injecting DL-homocysteic acid (DLH) into the ventral respiratory group (VRG). In intact rats, DLH produced tachypnea only when injected into the pre-Bötzinger complex (preBötC). Injections into pre-BötC and rostral VRG (rVRG) caused hypotension, whereas injections into the Bötzinger region elevated AP. Selective unilateral ablation of RVLM NK1Rimmunoreactive cells ( $97 \%$ loss within the pre-BötC and rVRG without loss of catecholaminergic neurons) was done by injecting saporin (SAP) conjugated with a selective NK1R agonist $\left[\mathrm{Sar}^{9}, \operatorname{Met}\left(\mathrm{O}_{2}\right)^{11}\right]$-substance $\mathrm{P}(\mathrm{SSP})$. Free SAP produced no
\end{abstract}

The pre-Bötzinger complex (pre-BötC) is a region of the ventral respiratory group ( $\mathrm{VRG}$ ) that is characterized in the adult by a distinctive mix of propriomedullary neurons with respiratory discharges (Ellenberger and Feldman, 1990; Connelly et al., 1992; Dobbins and Feldman, 1994; Schwarzacher et al., 1995). In neonate preparations in vitro, the pre-BötC is the main source of a respiratory-like rhythm that is transmitted polysynaptically to hypoglossal or phrenic motoneurons (Smith et al., 1991; Feldman and McCrimmon, 1999). This rhythm derives from a kernel of glutamatergic neurons that have intrinsic bursting properties and are synchronized by reciprocal recurrent excitation and, possibly, by gap junctions (Johnson et al., 1994; Butera et al., 1999a,b; Koshiya and Smith, 1999; Lieske et al., 2000; Rekling et al., 2000). On the basis of these studies, a hybrid-pacemaker-network theory of respiration has been proposed (for review, see Smith et al., 2000). This model postulates that rhythm generation is initiated by a cluster of bursting glutamatergic cells rather than by reciprocal interactions between two or more sets of inhibitory neurons (Duffin et al., 1995).

The adult characteristics of the pre-BötC glutamatergic neurons identified as rhythmogenic in the neonate are unknown, and their precise role in adult eupneic breathing remains speculative. Accordingly, the suggestion by Gray et al. (1999) that the neurokinin-1 receptor (NK1R) might be a marker of some form of excitatory pre-BötC neurons involved in respiratory rhythm

\footnotetext{
Received Nov. 29, 2001; revised Jan. 22, 2002; accepted Feb. 14, 2002.

This work was supported by National Institutes of Health Grants HL28785 and HL 60003 to P.G.G.

Correspondence should be addressed to Dr. Hong Wang, University of Virginia Health System, P.O. Box 800735, 1300 Jefferson Park Avenue, Charlottesville, VA 22908-0735. E-mail: hw8t@virginia.edu.

Copyright (C) 2002 Society for Neuroscience $\quad 0270-6474 / 02 / 223755-10 \$ 15.00 / 0$
}

lesion. Resting AP was normal in SAP- and SSP-SAP-treated rats, but the PND rate was slightly elevated in SSP-SAP-treated rats. The response of SAP-treated rats to DLH injection into VRG was normal and identical on each side, but tachypnea could not be elicited in the pre-BötC of SSP-SAP-treated rats on the toxin-injected side, and DLH caused a long-lasting apnea on the untreated side. The hypotension produced by DLH injection into pre-BötC and rVRG of SSP-SAP-treated rats was reduced on the lesioned side only.

In conclusion, NK1R-expressing cells of the rostral ventrolateral medulla control both respiratory rhythm and blood pressure. However, there is no evidence yet that these two functions are regulated by the same NK1R-expressing neurons.

Key words: substance P; Bötzinger; pre-Bötzinger complex; respiratory rhythm generation; blood pressure; saporin generation has stirred considerable interest (Pilowsky and Feldman, 2001). NK1R immunoreactivity is only detectable in a minority of VRG neurons that are not motoneurons and generally lack markers of inhibitory transmission (Liu et al., 2001; Pilowsky and Feldman, 2001; Wang et al., 2001). A specific type of pre-BötC inspiratory neuron of the adult rat was also shown to be strongly NK1R-immunoreactive (ir) (Guyenet and Wang, 2001) and the selective bilateral ablation of the NK1R-ir neurons of the pre-BötC produces major respiratory deficits in awake rats (Gray et al., 2001). Altogether these results suggest that some of the pre-BötC NK1R-ir neurons are an essential component of the VRG circuit that controls the respiratory rhythm. The aim of the present study is to test this hypothesis further and to determine whether some of the NK1R-expressing cells of the VRG also regulate arterial pressure.

A defining characteristic of the pre-BötC in vivo is the tachypneic response that can be produced by injecting excitatory amino acids at this level of the medulla (Chitravanshi and Sapru, 1999; Solomon et al., 1999). To test the contribution of the NK1R-ir neurons to the amino acid-induced tachypnea, we examined whether this response is modified after selective unilateral ablation of the NK1R-ir cells. Selective ablation of the NK1R-ir neurons was done by intraparenchymal injection of a conjugate of the ribosomal toxin saporin (SAP) with a selective NK1R agonist (Mantyh, 1997; Wiley and Lappi, 1999; Gray et al., 2001). We also examined the effects of these lesions on the amino acid-induced changes in arterial pressure to assess whether the NK1R-ir cells of the VRG also regulate sympathetic tone.

\section{MATERIALS AND METHODS}

This report describes results obtained in 17 male Sprague Dawley rats (250-350 gm; Hilltop Laboratories, Scottsdale, PA) in which all phases of 
the experiments were technically successful, including the accurate placement of lesions and DL-homocysteic acid (DLH) microinjections. An additional 15 rats were used to optimize the dose of [Sar ${ }^{9}$, Met $\left(\mathrm{O}_{2}\right)^{11}$ ]-substance P (SSP)-SAP. All experiments were performed in accordance with National Institutes of Health and institutional animal care and use guidelines. All procedures and protocols were approved by the University of Virginia's Animal Research Committee.

Lesion of NK1R-ir neurons in the VRG. The surgery was done using aseptic procedures. Anesthesia was induced with a mixture of ketamine (75 mg/kg), xylazine $(5 \mathrm{mg} / \mathrm{kg})$, and acepromazine $(1 \mathrm{mg} / \mathrm{kg})$ administered intramuscularly. Additional doses of anesthetic $(10 \%$ of initial dose) were given as needed. The saporin conjugate SSP-SAP or free SAP (both from Advanced Targeting Systems, San Diego, CA) was administered into the left ventrolateral medulla by pressure injection (PLI-100, Medical Systems Corp., Greenvale, NY) using glass micropipettes with a tip diameter of $25 \mu \mathrm{m}$. The head of each rat was fixed in a stereotaxic frame (David Kopf Instruments, Tujunga, CA) with the mouthpiece set at $-11 \mathrm{~mm}$ below the interaural line. The atlanto-occipital membrane was slit, and the lower aspect of the occipital plate was removed to allow penetration of the injection pipettes. Three $50 \mathrm{nl}$ injections $(0.313 \mathrm{ng}$ each in $0.9 \%$ sterile $\mathrm{NaCl}$ ) of either SSP-SAP (experimental group, $n=$ 5) or SAP (control group, $n=5$ ) were made into the ventrolateral medulla to target the entire VRG rostral to the lateral reticular nucleus. The coordinates of the injection sites were $0,0.5$, and $1 \mathrm{~mm}$, respectively, rostral to the obex, $1.9 \mathrm{~mm}$ lateral to the calamus scriptorius, and $2.4 \mathrm{~mm}$ below the dorsal surface of the medulla, with the electrode positioned at an angle of $30^{\circ}$ from the vertical pointing rostrally. After the surgery, the rats were treated with an antibiotic (ampicillin, $125 \mathrm{mg} / \mathrm{kg}$, i.m.; BristolMyers Squibb Company, Princeton, NJ) and an analgesic (ketorolac, 0.6 $\mathrm{mg} / \mathrm{kg}$ ) and then returned to standard housing conditions. Animals were allowed to survive 2-3 weeks before they were used for physiological experiments. The unilateral injections of toxin produced no observable behavioral effects.

The dose of SSP-SAP used in the present study $(0.313 \mathrm{ng} / 50 \mathrm{nl})$ was selected after experimenting with a much wider range of doses $(0.156-$ $2.5 \mathrm{ng}$ ) on 15 rats in which the tissue was processed for NK1R and tyrosine hydroxylase $(\mathrm{TH})$ immunoreactivity. The selected dose created optimal lesion of the NK1R-ir neurons of the VRG $(>95 \%)$ while preserving the integrity of the TH-ir cells. Doses 2-4 times higher created a visible necrosis at the injection center. Smaller doses produced only partial lesions of the NK1R-ir cells.

Physiological experiments. Recordings were made in three groups of rats: untreated controls $(n=7)$, SSP-SAP-treated $(n=5)$, and SAPtreated controls $(n=5)$. Anesthesia was induced with 5\% halothane in $100 \%$ oxygen and maintained at $1.6-1.8 \%$ during surgery via a tracheal cannula (60 cycles/min; $\sim 1 \mathrm{ml} / 100 \mathrm{gm}$ ). End-expiratory $\mathrm{CO}_{2}$ was monitored using infrared spectroscopy (Columbus Instruments, Columbus, $\mathrm{OH})$ and maintained at $4.5-5 \%$ during surgery. Rectal temperature was kept between 37.5 and $38.5^{\circ} \mathrm{C}$. The vagus nerves were cut bilaterally in the neck. A femoral artery and vein were catheterized to monitor arterial blood pressure (AP) and to administer drugs, respectively. The rats were placed in the stereotaxic frame with the mouthpiece set at $3.5 \mathrm{~mm}$ below the interaural line. The right phrenic nerve was placed on bipolar wire electrodes to record inspiratory activity. A concentric bipolar stimulating electrode (Rhodes Medical Instruments, Woodland, CA; diameter 250 $\mu \mathrm{m}$; tip separation $500 \mu \mathrm{m}$ ) was placed in the fascia surrounding the mandibular branch of the facial nerve on the right side of the rat. This electrode was used to locate the caudal pole of the facial motor nucleus by means of antidromic field potential recordings (monophasic square pulses; $100 \mu \mathrm{sec} ; 0.5-2 \mathrm{~mA} ; 1 \mathrm{~Hz}$ ) (Brown and Guyenet, 1985). After completion of surgery, halothane was replaced by urethane given at an initial dose of $1.0 \mathrm{gm} / \mathrm{kg}$, intravenously. After $1 \mathrm{hr}$ equilibration, the muscle relaxant pancuronium was administered $(1 \mathrm{mg} / \mathrm{kg}$, i.v.; with $0.3-0.5 \mathrm{mg} / \mathrm{kg}$ supplements as required), and electrophysiological recordings were initiated. Up to three supplements of $0.1 \mathrm{gm} / \mathrm{kg}$ urethane each were administered during the next $5 \mathrm{hr}$ such that the rise of AP after the delivery of a strong nociceptive stimulus to the foot or tail never exceeded $10 \mathrm{mmHg}$, and no change in the frequency or amplitude of the phrenic nerve discharge (PND) was caused by these stimuli. Ventilation was finally adjusted so that end-expiratory $\mathrm{CO}_{2}$ was $\sim 0.5 \%$ above the threshold of the phrenic discharge.

The PND was amplified, filtered $(200-3000 \mathrm{~Hz})$, full-wave rectified, and integrated with a sample-hold integrator with $50 \mathrm{msec}$ bins. All physiological variables (AP, end-expiratory $\mathrm{CO}_{2}, \mathrm{PND}$, integrated PND) were recorded on a computer through a Power 1401 interface and the
Spike2 software (version 3) (both from Cambridge Electronics Design Ltd., Cambridge, UK). For illustration, representative excerpts of the Spike2 data files were exported into a drawing program (Canvas 6, Deneba, Miami, FL).

Small pressure injections of the excitatory amino acid DLH (10 mM in $0.9 \%$ saline; $5-10 \mathrm{nl}$ delivered in $1 \mathrm{sec}$ through glass pipettes with an exterior tip diameter of $25 \mu \mathrm{m}$ ) were used to depolarize groups of neurons at specific sites in the medulla. Dose-effect relationships were not systematically investigated, but the selected dose of DLH proved reliably effective in increasing PND rate without causing significant tonic phrenic discharge. This dosage produced reproducible effects when injected twice in a row into the same site at a $5 \mathrm{~min}$ interval. Reproducibility across sites and animals was judged by the fact that characteristic site-dependent effects were produced. The DLH solution contained green fluorescent beads (Lumafluor Corp., Naples, FL; 1\% of the commercial solution by volume) that were used to locate the injection sites. The injections were made $1.9 \mathrm{~mm}$ lateral to the midline into various subdivisions of the VRG. The caudal end of the facial motor nucleus was used as a neurophysiological landmark to identify the stereotaxic location of the various subdivisions of the VRG. On the basis of our previous unit recording experiments (Guyenet and Wang, 2001; Wang et al., 2001), we estimate that the Bötzinger region is located just behind the facial motor nucleus at a depth corresponding to the bottom of the facial nucleus $\pm 150 \mu \mathrm{m}$ and extends from 0 to $600 \mu \mathrm{m}$ behind this structure. On the basis of the same series of recordings, the pre-Bötzinger region extends $\sim 600-1100 \mu \mathrm{m}$ caudal to the facial field, and the rostral VRG (rVRG) extends from 1100 to at least $1500 \mu \mathrm{m}$ caudal to it. Each respiratory subgroup is centered $1.9 \mathrm{~mm}$ lateral to the midline, and each subdivision is found at the same vertical coordinate in our preparation. After a preliminary mapping of the facial motor nucleus was accomplished, the recording electrode was withdrawn, it was replaced by an electrode containing DLH, and a longitudinal row of several microinjections of DLH were made into the ventral respiratory group always at the same lateral and depth coordinates. The rostrocaudal distance between injections varied according to the experimental group, and at least 5 min were allowed between consecutive injections. After a row of injections was completed on one side of the VRG, the injection pipette was moved symmetrically to the contralateral side of the VRG, and the same number of microinjections was performed into symmetrical locations in the contralateral side of the VRG by moving the electrode $3.8 \mathrm{~mm}$ toward the contralateral side.

The effect of DLH on each dependent variable (AP, PND rate, and amplitude) was expressed as a percentage change relative to baseline. Baseline respiratory rate and amplitude were determined by averaging 10 consecutive respiratory cycles immediately before the DLH injection, and the baseline value of mean AP (MAP) was also computed during the same $\sim 10 \mathrm{sec}$ period. The maximal change in PND rate occurred immediately after the injections of DLH. Except when noted in Results, the effect of DLH on rate was measured by averaging the period of 10 consecutive respiratory cycles immediately after microinjection of DLH. The maximum effect of DLH microinjection on AP was determined by averaging AP during a $5 \mathrm{sec}$ period centered on the nadir or apex of the response as appropriate.

Histology. At the end of the experiment, the rats were deeply anesthetized with additional urethane $(0.5 \mathrm{gm} / \mathrm{kg})$ and perfused transcardially with $250 \mathrm{ml}$ of PBS, pH 7.4, followed by $500 \mathrm{ml}$ of $4 \%$ phosphate-buffered (0.1 M, pH 7.35) formaldehyde (Fisher Scientific, Pittsburgh, PA). The brainstem was post-fixed overnight with the same fixative at $4^{\circ} \mathrm{C}$. Coronal sections $(30 \mu \mathrm{m})$ were cut through the medulla using a Vibratome (Lancer; Ted Pella, Inc., Redding, CA). One set of sections from a series of one in six (180 $\mu \mathrm{m}$ apart) was mounted and air dried, and coverslips were affixed with Krystalon (EM Industrial, Inc., Gibbstown, NJ). These sections were immediately examined under fluorescence to ascertain whether the injection sites were correctly placed. Animals in which the injection sites were incorrect were analyzed no further. The remaining sections were stored in cryoprotectant solution at $-20^{\circ} \mathrm{C}$. After up to 2 weeks, a second set of one in six sections was used for simultaneous immunohistochemical detection of $\mathrm{TH}$ and NK1Rs using previously described methods (Wang et al., 2001). The following primary antibodies were used: rabbit polyclonal antibody against NK1R (Chemicon International, Temecula, CA; 1:2000) and mouse monoclonal antiserum against TH (Chemicon International; 1:2000). The following secondary antibodies diluted at 1:200 were used: $\mathrm{F}\left(\mathrm{ab}^{\prime}\right)_{2}$ fragment goat anti-rabbit IgG conjugated to $\mathrm{Cy} 3$ (Jackson ImmunoResearch Laboratories, Inc., West Grove, PA) and goat anti-mouse IgG conjugated to Alexa 488 
A
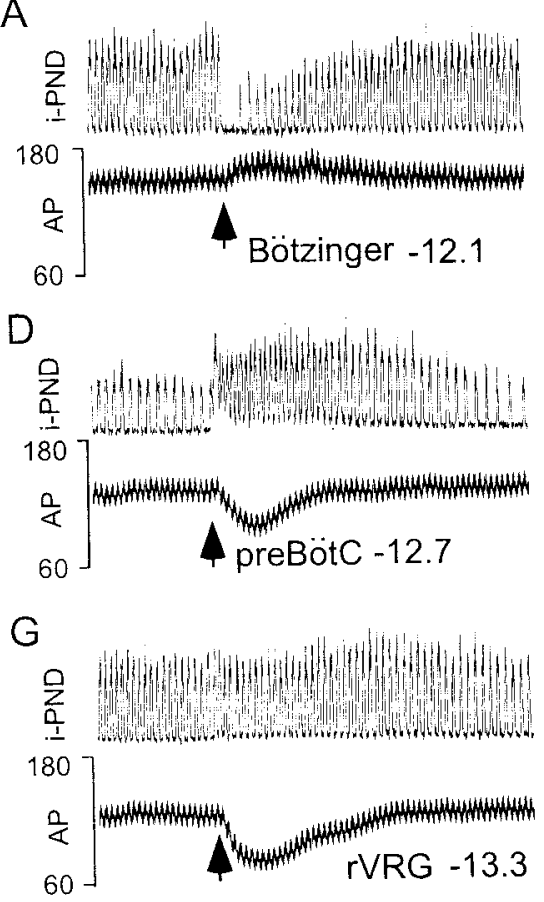

B

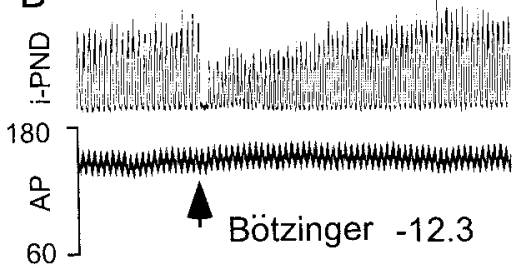

$\mathrm{E}$
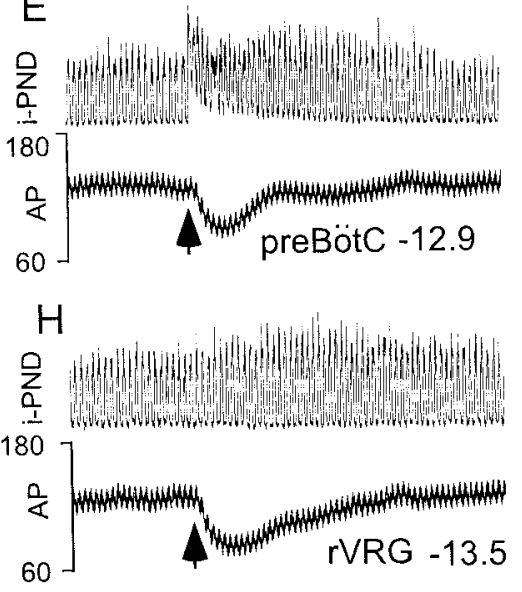

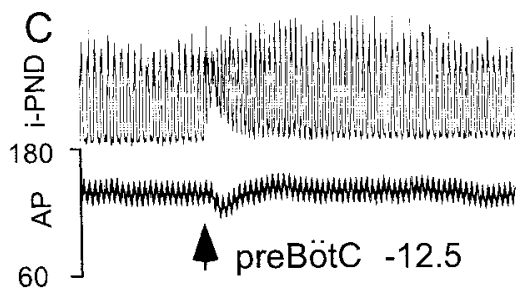

$\mathrm{F}$

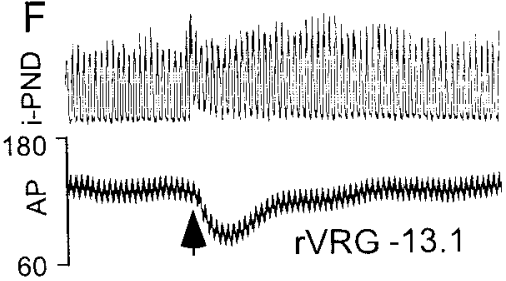

$\overline{10 s}$

Figure 1. Cardiorespiratory responses to microinjection of DLH into the VRG of intact rats. DLH was microinjected into the VRG at intervals of 200 $\mu \mathrm{m}$ at the time indicated by the arrows. $i$ - $P N D$, Integrated phrenic nerve charge (vertical scale arbitrary); $A P$, mean arterial pressure (scale in mmHg). The numbers under the AP traces indicate the rostrocaudal level of each injection relative to bregma. All records are from the same rat.

(1:200; Molecular Probes, Inc., Eugene, OR). The sections were mounted in sequential rostrocaudal order onto gelatin-coated slides, dehydrated, and briefly delipidated through graded alcohols and xylenes. Finally, coverslips were affixed with DPX mounting medium (Aldrich, Milwaukee, WI). No immunoreactivity was observed in the absence of the primary antibodies (Wang et al., 2001).

Mapping and imaging. An ordered set of $30 \mu \mathrm{m}$ sections (one in six) was examined under dark-field illumination to identify the two sections that contained our chosen diagnostic landmarks (Wang et al., 2001). The caudal end of the facial motor nucleus was assigned the level $11.7 \mathrm{~mm}$ behind bregma according to the nomenclature of Paxinos and Watson (1998). The rostral end of the lateral reticular nucleus, where this structure displays a lateral and a medial portion, was assigned the level $13.0 \mathrm{~mm}$ behind bregma according to the same authors. The rest of the sections were assigned a bregma level determined arithmetically by their location relative to the two landmark sections, given that each section was separated by $180 \mu \mathrm{m}$.

Sections were then examined with a Leitz epifluorescence microscope. Alexa 488 and Cy3 could be visualized without interference from each other. The section outlines, major landmarks, and the location of the cells of interest were drawn or plotted using a Lucivid camera (MicroBrightfield, Colchester, VT) and a motor-driven microscope stage (Ludl Electronic Products, Hawthorne, NY) controlled by the Neurolucida software (MicroBrightfield) as described previously (Stornetta and Guyenet, 1999). The cell nucleus was clearly visible in cells immunoreactive for $\mathrm{TH}$, and only cell profiles that included a visible nucleus were counted. This procedure was not possible in the case of NK1R-ir neurons in which immunoreactivity lined the somatic and dendritic membranes. NK1R-ir profiles were counted as cells only when a soma of at least 10 $\mu \mathrm{m}$ in diameter contiguous with two or more primary dendrites was present. No double counting was possible because the mapped sections were at least $180 \mu \mathrm{m}$ apart. The Neurolucida files were exported to the Canvas software drawing program (Deneba) for final modifications and printing.

Representative photographs of the fluorescent neurons labeled with Alexa 488 and Cy3 were taken using a two-color Olympus BX50 WI confocal microscope equipped with Krypton and Argon lasers. The images were scanned through $10 \times$ objectives, acquired at a resolution of $1024 \times 1024$ pixels, and stored in 24-bit TIFF format. TIFF files were imported into Adobe Photoshop (version 5.0.1; Adobe Systems, Moun- tain View, CA). Multiple photomicrographs were assembled so that the figure fit the page (minimum resolution of 300 pixels/inch). The output levels of each panel were adjusted against the range of levels containing pixels. Contrast and brightness were also individually adjusted to best reflect the original images. Lettering, scale bars (taking into account the final image size and resolution), and arrows were then added, also in Photoshop.

Statistical analyses. All data are presented as means \pm SEM. Differences between groups were evaluated with one-way ANOVA or two-way repeated measure ANOVA (RM ANOVA) as required (SigmaStat, Jandel Scientific, San Rafael, CA), except for the main experiment (see Fig. 5), which demanded more complex statistical treatment. In this case, an RM ANOVA procedure was performed to analyze experimental effects on blood pressure or PND. The two response variables and effects on these variables were regarded as independent. Treatment (SSP-SAP or SAP) was the between-subject variable in each of the models. Side (treated vs untreated) and location of injections (Bötzinger vs pre-BötC vs rVRG) were defined as repeated measure variables. Compound symmetry was assumed for the covariance structure of location, and an unstructured covariance structure was assumed for side in the Kronecker product structure for the bivariate repeated measures. Each repeated measure model included the three-way interaction for treatment-sidelocation, the three two-way interactions for treatment-side, treatmentlocation, and location-side, as well as the main effects. Twelve pair-wise comparisons were specified in advance of the experiment for each model. The difference of least squares means between the left and right sides in each experimental group at each location was evaluated. The least squares means for experimental and sham groups were also compared at each side in each location. The conservative Bonferroni multiple comparisons adjustment was applied to these contrasts. Significance level was set at 0.05 .

\section{RESULTS}

\section{Cardiorespiratory responses to microinjection of DLH in intact rats}

Figure 1 depicts the typical effect of a longitudinally oriented series of DLH injections into the VRG (injections made at 200 $\mu \mathrm{m}$ intervals). The same procedure was repeated in six other rats 

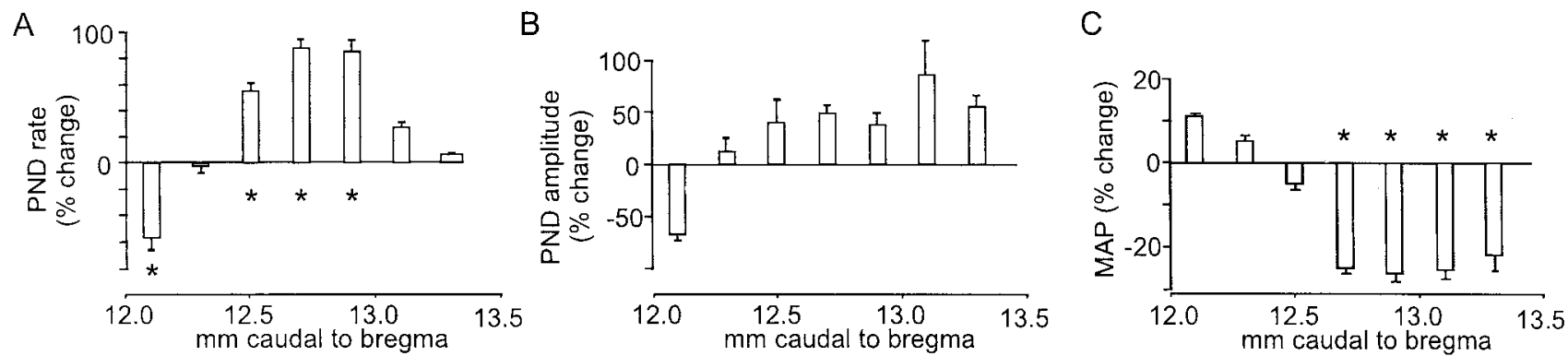

Figure 2. Cardiorespiratory responses to microinjection of DLH into the VRG of intact rats: group data. The peak effects of DLH on each of the dependent variables are expressed as a percentage change from the baseline values recorded during 10 sec before DLH injection. $A$, Effect on PND rate $(F=9.73 ; p<0.0001$ by one-way RM ANOVA $) . B$, Effect on PND amplitude $(F=1.46$; NS by one-way RM ANOVA $)$. $C$, Effect on MAP $(F=14.5 ;$ $p<0.001$ by one-way RM ANOVA). $n=7$ rats; five to seven sites injected per rat. ${ }^{*} p<0.05$ (least square means comparisons against zero).

with similar results. Figure 2 illustrates the average effect of these injections on the three dependent variables measured (PND rate, PND amplitude, and MAP). The effect of DLH on cardiorespiratory parameters was site specific. At rostral levels (bregma $-12.1 \mathrm{~mm}$ ), DLH produced a brief apnea followed by a slower PND rate and smaller PND amplitude. This response was associated with a small but consistent rise in AP. At more caudal levels, the bradypnea was gradually replaced by a tachypnea. The tachypnea was accompanied by a modest increase in PND amplitude and a drop in AP. The effects of DLH on PND rate and AP reversed direction at approximately the same cross-over point (bregma -12.3 to $-12.4 \mathrm{~mm}$ ) (Fig. $2 A-C$ ). Caudal to a point situated $\sim 13.1 \mathrm{~mm}$ behind bregma, the tachypneic response disappeared, whereas the drop in blood pressure persisted (Figs. $1 G, H, 2 A-C)$. DLH injection into this area also generally increased PND amplitude, although the response was variable and the overall effect was not significant (Fig. $2 B$ ). To exclude the possibility that some of the respiratory responses to DLH could have been secondary to the drop in AP, sodium nitroprusside (5 $\mu \mathrm{g} / \mathrm{kg}$, i.v.) was administered in two rats at a dose that produced the same decrease in MAP. Sodium nitroprusside produced no detectable effect on PND (data not shown). In each animal the injection sites were identified by the presence of fluorescence microbeads. The location of the microbeads at three representative levels of the VRG is illustrated in Figure $3 A-C$. All injection sites were located ventral to the nucleus ambiguus in a narrow column of the ventrolateral medulla that corresponds to the VRG.

\section{Cardiorespiratory responses to microinjection of DLH in rats with unilateral injections of SSP-SAP conjugate or free SAP}

Two groups of rats were compared. An experimental group received unilateral injections of SSP-SAP into the VRG and a control group received SAP instead. The physiological experiments were performed 14-21 d after this injection procedure. In each rat, DLH was microinjected into three specific portions of the VRG, namely the Bötzinger region (two injections at 12.1 and $12.3 \mathrm{~mm}$ behind bregma), the pre-BötC (two injections at 12.7 and $12.9 \mathrm{~mm}$ behind bregma), and the rVRG (two injections at 13.1 and $13.3 \mathrm{~mm}$ behind bregma). The effect of these pairs of injections was pooled, generating one data point per site for each dependent variable. The procedure was then repeated on the other side of the brain. The following results are from experiments in which DLH microinjections were on target on both sides of the brain, and full histological documentation of the effect of the lesion was obtained. The histological data will be described in the next section.

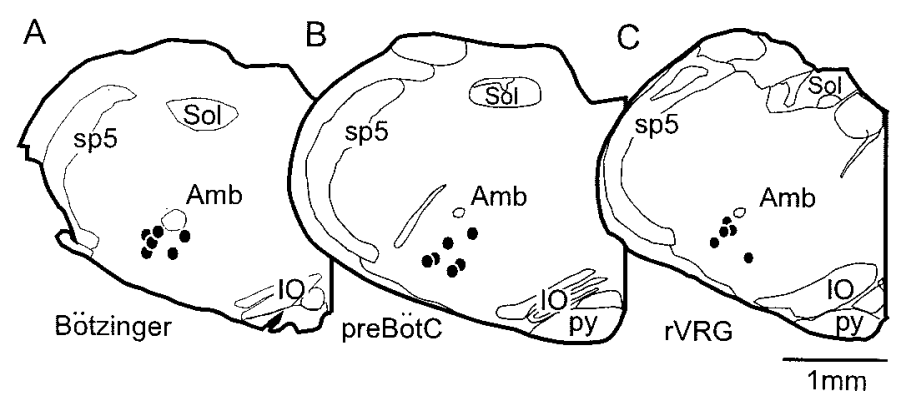

Figure 3. DLH injection sites at three levels of the VRG. Injection sites were marked by fluorescein-tagged microbeads (see Fig. 7), and their location was plotted with a computer-controlled microscope stage and drawing program. The locations of the microbeads found at levels corresponding to the Bötzinger region (bregma $-12.1 \mathrm{~mm} ; 7$ sites), the preBötC ( $-12.7 \mathrm{~mm}$; 6 sites), and the $\mathrm{rVRG}(-13.1 \mathrm{~mm}, 5$ sites $)$ are represented. The sites are from the seven animals described in Figure 2. Two rats did not receive DLH injections at each of the three levels represented. $A m b$, Nucleus ambiguus pars compacta; IO, inferior olive; pyr, pyramidal tract; Sol, nucleus of the solitary tract; sp5, spinal trigeminal tract.

The resting cardiovascular parameters of SSP-SAP- and SAPtreated rats were generally no different from that of rats that had not been treated previously (Table 1). One exception was the basal PND rate, which was slightly higher in the SSP-SAP-treated group (Table 1).

In SAP-treated rats, DLH injection produced the same sitedependent cardiorespiratory changes as in previously untreated rats. Figure 4 illustrates a representative case. The group data (Fig. $5 A, B)$ indicate that DLH injection produced identical effects on each side of the brain. In these experiments, we did not analyze the effects of DLH on PND amplitude because these effects were weak and rather variable everywhere in the VRG except in the Bötzinger region.

In SSP-SAP-treated rats, the DLH injection produced distinctly different effects that are illustrated by one representative case (Fig. 6) and the group data of Figure 5, $C$ and $D$. The changes were site specific. Although the cardiorespiratory response to DLH injection into the Bötzinger region was not significantly affected (compare Figs. $4 A 1$ and $6 A 1$; for summary see Fig. 5), several changes were observed at the pre-BötC level. On the toxin-treated side, the tachypnea observed in control rats was replaced by a mild bradypnea and minor reduction in PND amplitude (compare Fig. $6 A 2$ with Figs. $1 D$ or $4 A 2$; Fig. 5 ). On the untreated side of the SSP-SAP-treated rats, the phrenic response to DLH injection was also abnormal but in a different way. 
Table 1. Resting cardiorespiratory variables in the three groups of rats

\begin{tabular}{llll} 
Group & $\begin{array}{l}\mathrm{MAP} \\
(\mathrm{mmHg})\end{array}$ & $\begin{array}{l}\text { PND rate } \\
(\text { bursts } / \mathrm{min})\end{array}$ & $\mathrm{CO}_{2}(\%)$ \\
\hline Untreated $(n=7)$ & $141.6 \pm 2.0$ & $45.5 \pm 1.7$ & $3.0 \pm 0.1$ \\
SAP treated $(n=5)$ & $134.6 \pm 1.9$ & $47.9 \pm 1.8$ & $2.7 \pm 0.1$ \\
SSP-SAP treated $(n=5)$ & $124.2 \pm 6.3$ & $69.1 \pm 3.5^{*}$ & $2.6 \pm 0.1$ \\
\hline
\end{tabular}

${ }^{*} p<0.05$ when compared with SAP-treated rats and untreated rats (ANOVA).

DLH produced a very brief series of high-frequency respiratory bursts (Fig. 6B2) that were immediately followed by an extremely long period of phrenic apnea (Fig. 6B2, inset). In other rats (three of five), the long period of apnea occurred immediately after DLH injection and was followed by tonic activity preceding resumption of a normal phrenic discharge (data not illustrated). Finally, the AP drop produced by DLH injection into the preBötC and rVRG of SSP-SAP-treated rats was significantly reduced on the treated side only (Figs. $5 C, 6 A 2-A 3$ ). The hypotension produced by DLH on the untreated side of the SSP-SAPtreated rats was identical to that observed in the SAP-treated control group (Figs. 5C, 6B1-B3).

\section{DLH injection sites and NK1R-ir neurons in rats treated with SSP-SAP or SAP}

Unilateral injection of unconjuguated SAP produced no effect on the VRG as judged by the integrity of NK1R-ir and TH-ir neurons on both sides of the VRG (Fig. $7 A, B$ ). Unilateral injection of SSP-SAP eliminated NK1R-ir neurons on the treated side but spared the TH-ir cells (Fig. $7 C$ ). On the contralateral (untreated) side, NK1R-ir and TH-ir neurons were both apparently intact (Fig. 7D).

The entire distribution of NK1R-ir neurons was plotted at levels corresponding to the Bötzinger $(-11.9 \mathrm{~mm})$, pre-BötC $(-12.6 \mathrm{~mm})$, and $\mathrm{rVRG}(-13.4 \mathrm{~mm})$ in a representative SSPSAP-treated rat (Fig. 8). As shown previously (Nakaya et al., 1994), NK1R-ir neurons were widely distributed in the rostral medulla oblongata with particular concentrations in the vestibular nuclei, nucleus of the solitary tract, ventromedial medulla, intermediate reticular formation, and ventrolateral medulla. As shown previously (Wang et al., 2001), even within the ventrolateral medulla, NK1R-ir neurons are not confined to the VRG (region outlined by the $500 \times 500 \mu \mathrm{m}$ square), although their number is especially high within the pre-BötC region of the VRG (Fig. $8 B$ ). As shown in Figure 8, SSP-SAP treatment eliminated virtually all NK1R-ir neurons of the ventrolateral medulla from bregma $-11.9 \mathrm{~mm}$ (200 $\mu \mathrm{m}$ caudal to the facial motonucleus) to the level of the rVRG. In every instance the most rostral portion of the nucleus ambiguus (pars compacta) that overlies the Bötzinger region was intact, whereas more caudal aspects of the same nucleus were partially destroyed. The NK1R-ir neurons located in the ventromedial medulla dorsal to the olive were intact even on the ipsilateral side, as were the NK1R-ir neurons of the nucleus of the solitary tract and those of the intermediate reticular formation.

For quantitative purposes, we counted systematically the number of NK1R-ir and TH-ir cells located in the VRG of the five SSP-SAP-treated rats. Counts were made throughout the entire relevant portion of the VRG (bregma -11.7 to $-13.7 \mathrm{~mm}$ ) within the box shown in Figure 8. As shown in Figure 9A, NK1R-ir cells were indeed almost totally eliminated from all three subdivisions of the VRG on the SSP-SAP-treated side except at the most rostral level ( $-11.7 \mathrm{~mm}$ corresponding to the very caudal end of the facial motor nucleus). In contrast, the number of TH-ir cells was the same on both sides within the counted area. The number of NK1R-ir neurons was normal within the VRG on the side contralateral to the SSP-SAP injections (Wang et al., 2001). To further document the fact that SSP-SAP spared the NK1R-ir neurons on the uninjected side, we compared the number of NK1R-ir neurons present in the VRG of SSP-SAP-treated rats with the number found in the SAP-treated control rats. Counts of NK1R-ir neurons and TH-ir neurons were made at the level of the pre-BötC because this level corresponds to the geometric center of the toxin injections (two sections per rat; bregma level -12.7 and $-12.9 \mathrm{~mm}$ ), and this level should have been exposed to the highest toxin level on the injected side. The number of pre-BötC NK1R-ir cells on the side contralateral to the SSP-SAP injections was exactly the same as on either side of the rats that had received unconjuguated SAP (Table 2). This observation strongly suggests that unilateral injection of SSP-SAP destroyed NK1R-ir neurons selectively on the treated side.

A computer-assisted plot of the pre-BötC DLH injection sites in the five control and experimental rats is shown in Figure 10. We could not detect a difference in the location of the injection sites either when left versus right sites were compared or when the injections sites of SSP-SAP-treated rats were compared with those of the control SAP-treated rats. Injection sites within the Bötzinger and rVRG were also plotted and found in the expected locations (data not shown).

\section{DISCUSSION}

According to the present results, NK1R-expressing cells located within the ventrolateral medulla control both respiratory rhythm and blood pressure. Whether the same or different neurons control both functions remains uncertain.

\section{Cardiorespiratory effects of DLH injection into the VRG}

As shown before, the chemical depolarization of VRG neurons with the amino acid DLH produces site-specific effects on blood pressure and respiration (Willette et al., 1987; Chitravanshi and Sapru, 1999; Solomon et al., 1999). Tachypnea was produced only when DLH was injected within a narrow rostrocaudal range of the VRG. This region [bregma $-12.3-13.1 \mathrm{~mm}$ according Paxinos and Watson (1998)] corresponds closely to the classically described pre-BötC (Ellenberger and Feldman, 1990; Smith et al., 1991; Connelly et al., 1992; Dobbins and Feldman, 1994; Schwarzacher et al., 1995). Rostral to it, DLH decreased PND amplitude and rate as reported before (Chitravanshi and Sapru, 1999) perhaps because of the activation of the inhibitory expiratory augmenting neurons of the Bötzinger region (Duffin et al., 1995; Schreihofer et al., 1999). Caudal to the pre-BötC, DLH did not change respiratory rate (Chitravanshi and Sapru, 1999), in keeping with the view that the rVRG is not involved in rhythmogenesis (Feldman and McCrimmon, 1999).

The sites where DLH injection produced tachypnea were located in the region of the VRG that contains a high density of NK1R-ir neurons [12.5-13 mm caudal to bregma according to Paxinos and Watson (1998)]. However, the intensity of the DLHinduced tachypnea and the density of NK1R-ir cells in the VRG were not tightly correlated. Indeed, substantial numbers of NK1R-ir cells are still present in the VRG both rostral (12-12.5 mm behind bregma) and caudal (13-13.5 mm behind bregma) to the hot spot for inducing tachypnea (Wang et al., 2001). Two possibilities can explain the discrepancy. The population of 

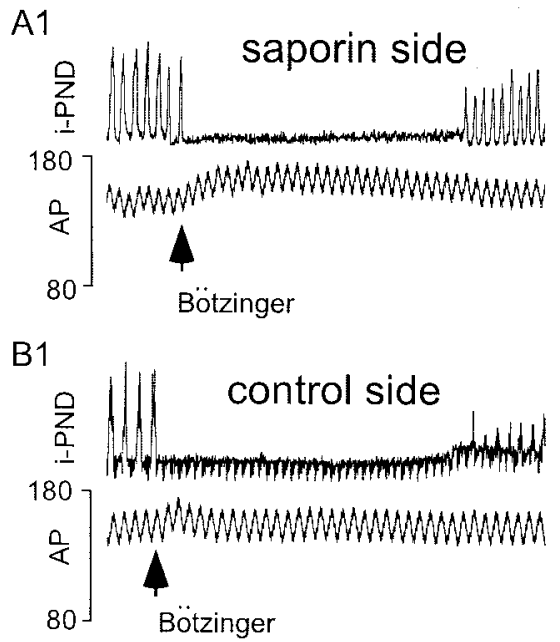

A2

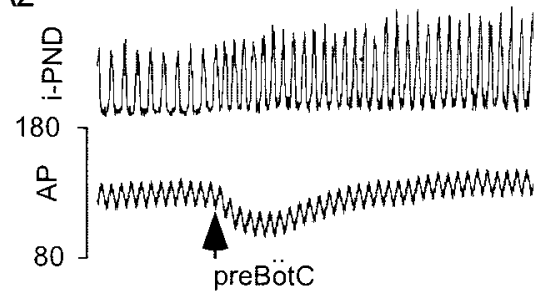

B2

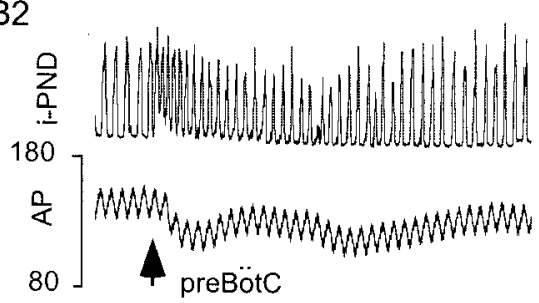

A3

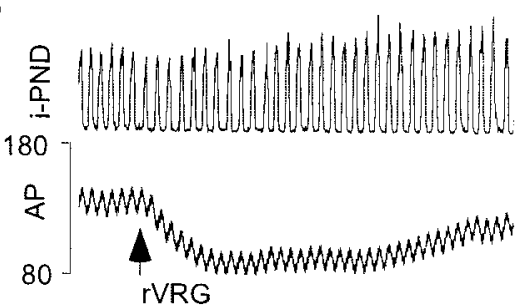

B3

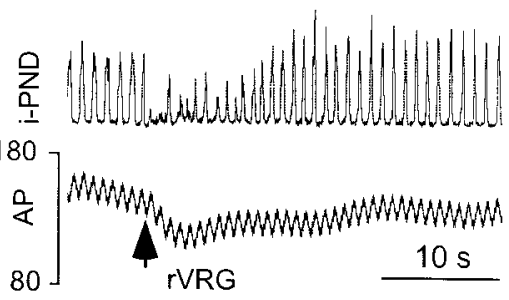

Figure 4. Effect of DLH injections into the VRG of a control SAP-treated rat. $A 1-A 3$, DLH injection into the left, SAP-treated side. B1-B3, DLH injections into the right (uninjected) side. All excerpts are from the same rat. $i-P N D$, Integrated PND (arbitrary vertical scale); AP is in mmHg. DLH injections are at arrows. As illustrated here, the effects of DLH were similar on both sides within the Bötzinger and pre-BötC. DLH injection into rV RG occasionally produced brief inhibition of PND amplitude.

A
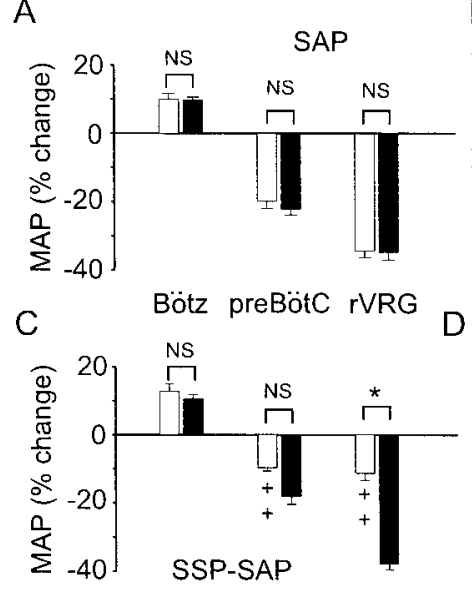

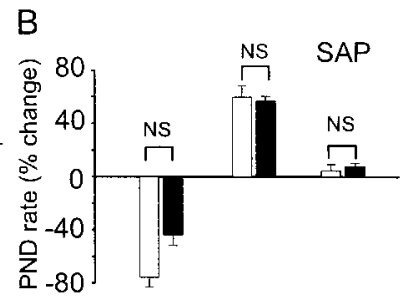

Bötz preBötC rVRG

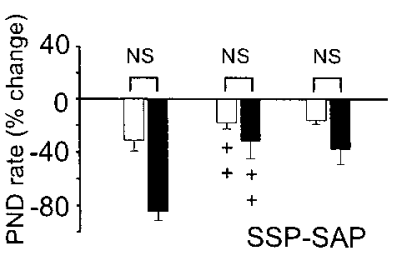

Figure 5. Effect of a unilateral destruction of NK1R-ir neurons on the cardiorespiratory responses to DLH injection into three regions of the VRG: summary data. In each graph, the open bars represent the effect of DLH on the left side of the brain that was treated either with SAP $(A, B)$ or with SSP-SAP $(C, D)$. Closed bars represent the effect of DLH on the opposite and untreated side. $A$, Peak effect of DLH on the BP of rats treated unilaterally with SAP ( $n=5$; control rats). $B$, Peak effect of DLH on PND rate in the same five control rats. $C$, Peak effect of DLH on the $\mathrm{BP}$ of rats treated unilaterally with SSP-SAP $(n=5$; experimental rats). $D$, Peak effect of DLH on PND rate in the same five experimental rats. A significant three-way interaction for treatment-side-location $(p=0.0199)$ was observed in the repeated measure model for blood pressure, demonstrating a complex treatment effect. Other lower order effects could not be easily interpreted because the three-way interaction was significant. Significant two-way interactions for treatment-side $(p=0.0534)$ and treatmentlocation $(p=0.0142)$ were obtained in the overall model for PND. The results of the most relevant post hoc pair-wise comparisons are indicated in the figure. Brackets refer to right versus left side within-group comparisons $\left({ }^{*} p<0.05\right) . \ddagger$ indicates significance at the 0.05 level for inter-group comparisons; e.g., left pre-BötC DLH injection in the control group versus left pre-BötC DLH injection in the experimental group.

NK1R-ir cells of the VRG may be functionally heterogeneous, and only a subpopulation may mediate the PND rate increase caused by amino acid injection. Alternatively, at some levels, especially in the Bötzinger region, the tachypnea produced by

activating the NK1R-ir neurons could be negated by the simultaneous activation of neurons that have opposite effects on respiratory rate.

Consistent changes of arterial pressure were associated with the respiratory effects. Because the animals were vagotomized, these cardiovascular effects almost certainly reflected changes in sympathetic nerve discharge. The cross-over point where pressor responses were replaced by hypotension corresponds to where the density of bulbospinal sympathoexcitatory neurons decreases and GABAergic interneurons thought to inhibit the former become more prevalent (Chan and Sawchenko, 1998; Schreihofer et al., 2000). In the cat, DLH microinjection into the VRG seems to cause inconsistent effects on MAP (Solomon et al., 1999). Differences in brain size and organization or injection sites may explain the discrepancy. By design, we targeted sites that were clearly ventral to the nucleus ambiguus to deposit the DLH as close as possible to the NK1R-expressing cells of the VRG. Thus our injection sites overlapped substantially with the blood pressure-regulating part of the ventrolateral medulla [RVLM and caudal ventrolateral medulla (CVLM), regions identified by the presence of catecholaminergic cells] (Willette et al., 1987; Dampney, 1994; Schreihofer and Guyenet, 1997).

\section{Selectivity of the lesions produced by SSP-SAP}

SSP-SAP destroys NK1R-expressing neurons by allowing the ribosomal toxin saporin to be internalized along with agonistbound NK1 receptors (Wiley and Lappi, 1999). Because SSP has very low affinity for other tachykinin receptors (Tousignant et al., 1990), its saporin conjuguate has little potential to damage cells that express tachykinin receptors other than NK1. Accordingly, the conjuguate destroyed virtually all NK1R-ir neurons of the VRG and spared the intermingled TH-ir neurons that do not express detectable levels of NK1R (Gray et al., 1999; Wang et al., 2001). Also, free saporin did not lesion NK1R-ir neurons, and saporin-treated rats were physiologically indistinguishable from untreated rats. Interestingly, the destruction of the NK1R-ir neurons of the VRG remained confined to the toxin-treated side (Table 2, Fig. 8), although these neurons project massively to the other side (Wang et al., 2001). Conceivably, these NK1R-ir cells do not have receptors on their axons and axonal terminals. Alterna- 


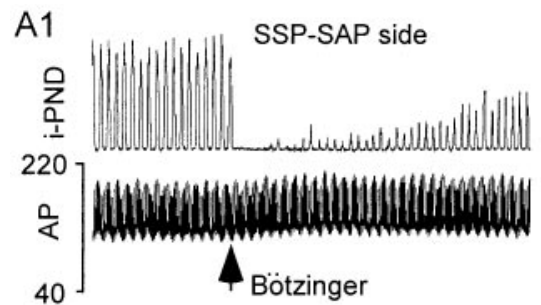

B1

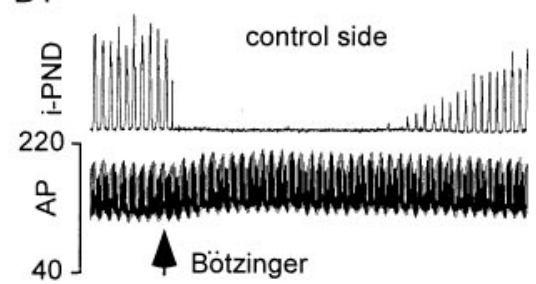

A2

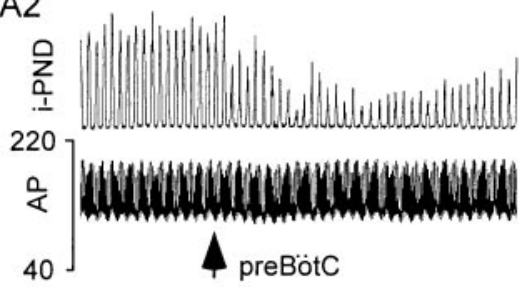

B2

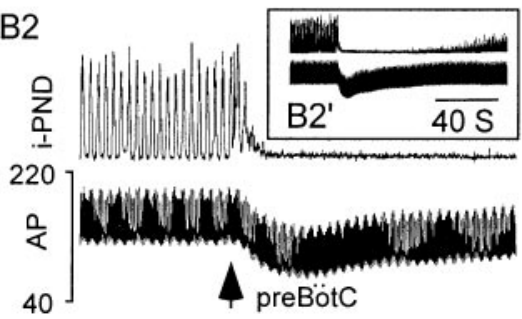

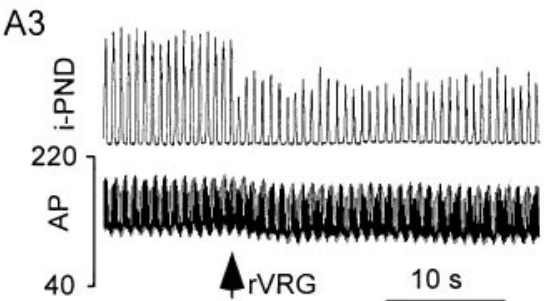

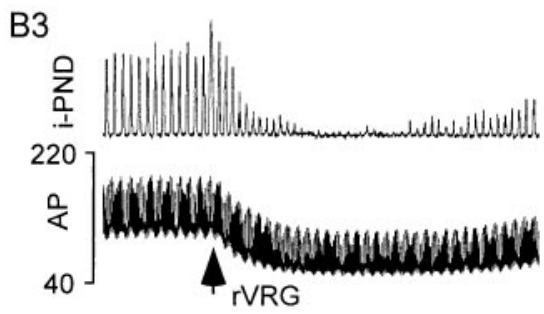

Figure 6. Effect of DLH injections into the VRG of a SSP-SAP-treated rat. $A 1-A 3$, DLH injection into the left, SSP-SAP-treated side. $B 1-B 3$, DLH injections into the right, untreated side. All excerpts are from the same rat. $i$-PND, Integrated PND (arbitrary vertical scale); AP is in mmHg. DLH injections are at arrows. The effects of DLH injection into the pre-BötC are abnormal on both sides. Inset B2' illustrates the long phrenic apnea caused by DLH on the untreated side where NK1R-ir cells were intact (note different time scale).
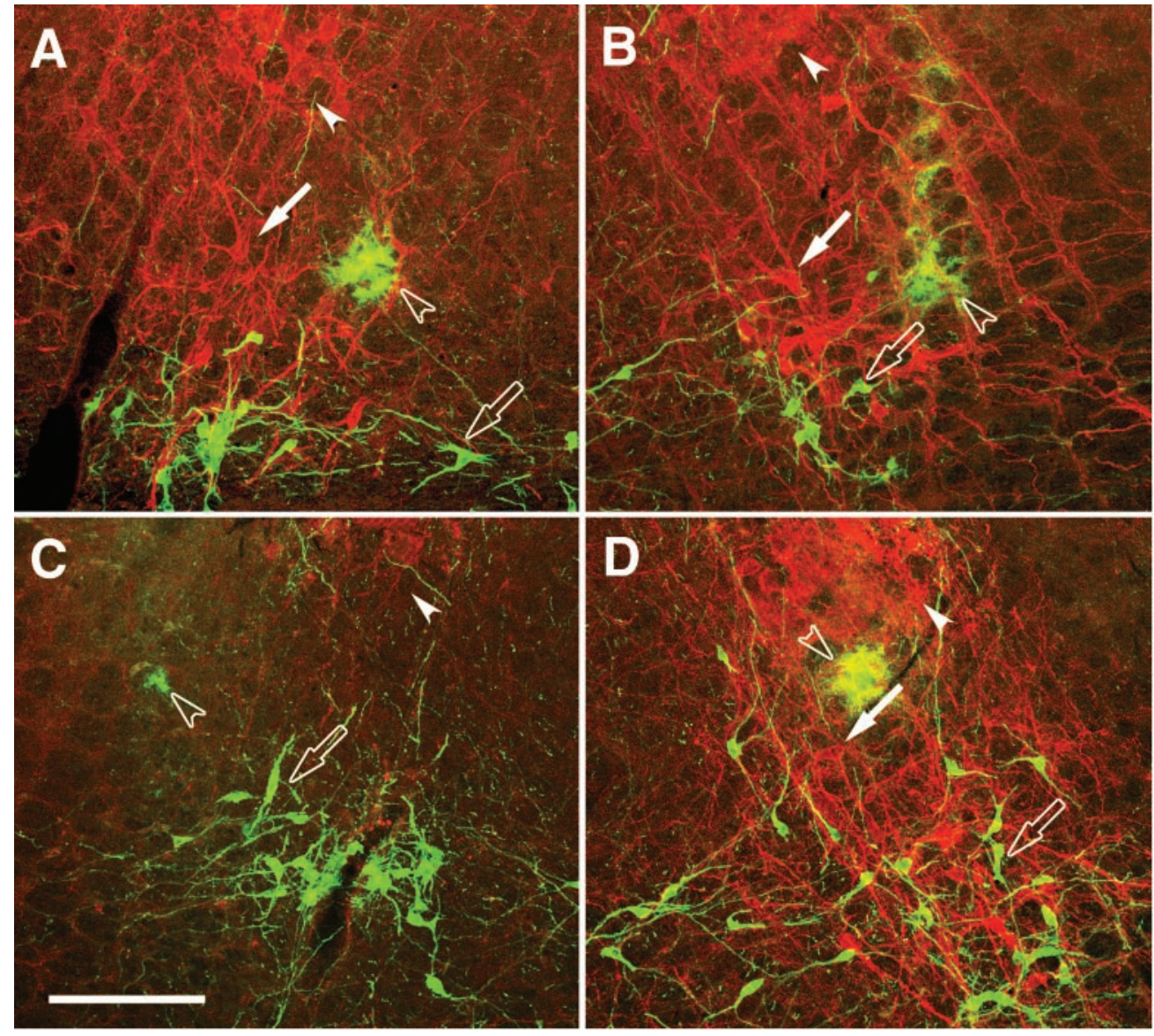

Figure 7. Selective lesion of NK1R-ir neurons with SSP-SAP. Confocal images of the pre-BötC in a control rat treated unilaterally with SAP ( $A$, treated side; $B$, untreated side) or in an experimental rat treated with SSP-SAP $(C$, treated side; $D$, untreated side) are shown. Alexa-488labeled TH-ir neurons are in green (open arrows); Cy-3 labeled NK1R-ir neurons are in red (white arrows). Each panel contains a deposit of fluorescent microbeads indicating where DLH injections have been placed in these animals during the course of the physiological experiments (open arrowheads). The pre-BötC lies between nucleus ambiguus (white arrowheads) and the TH-ir neurons and can be identified by its high density of NK1R-ir neurons (white arrows). Scale bar, $200 \mu \mathrm{m}$. tively, binding of SSP-SAP to presynaptic receptors may not trigger internalization, or saporin is not retrogradely transported to the cell bodies after internalization. The latter possibility is unlikely because saporin conjuguated to an antibody against dopamine $\beta$-hydroxylase destroys noradrenergic neurons after con- tact with distant projections of these neurons (Schreihofer et al., 2000). In sum, it remains possible that the toxin could destroy some NK1R-expressing neurons that send axons to or through the VRG, but this hypothetical population does not include the NK1R-ir neurons of the contralateral VRG. 


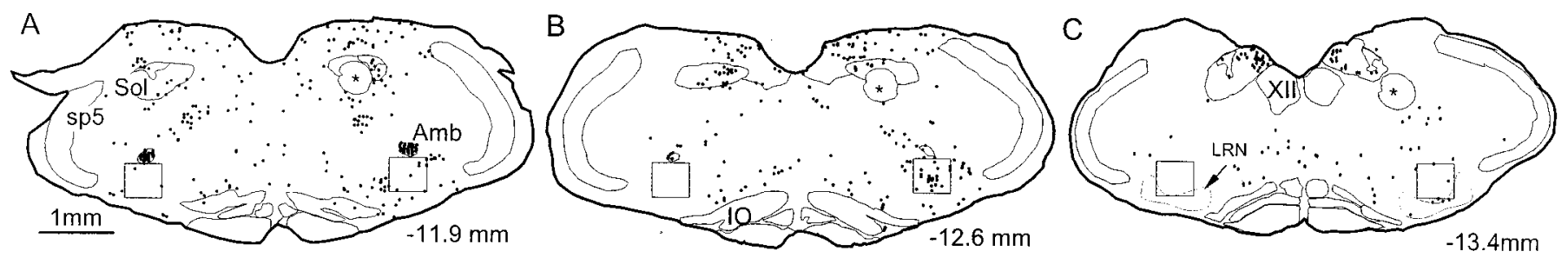

Figure 8. Distribution of NK1R-ir neurons in an SSP-SAP-injected rat. Computer-assisted plots of NK1R-ir neurons at three levels of the medulla oblongata at the level of the Bötzinger nucleus $(A)$, pre-BötC $(B)$, and $\operatorname{rVRG}(C)$ are shown. In each section a $500 \times 500 \mu \mathrm{m}$ box outlining the VRG is represented. SSP-SAP was injected on the left side. The star identifies a needle punch used to mark the right side of the sections. Amb, Nucleus ambiguus pars compacta; $I O$, inferior olive; $L R N$, lateral reticular nucleus; Sol, nucleus of the solitary tract; sp5, spinal trigeminal tract; XII, hypoglossal nucleus.

A

Figure 9. Rostrocaudal extent of the lesions. NK1R-ir and TH-ir neurons were counted in a $1 / 6$ series of $30-\mu \mathrm{m}$-thick coronal sections in the five rats treated unilaterally with SSP-SAP. Cells were counted on each side (in $A$ and $B$, the open circles represent the numbers on the untreated side; the closed circles represent the numbers on the treated side) within the $500 \times$ $500 \mu \mathrm{m}$ square box outlined in Figure 8. The square box was positioned so that the middle of its top side touched the bottom of the nucleus ambiguus. ${ }^{*} p<0.05$ (one-way RM ANOVA and Bonferroni pair-wise comparisons).

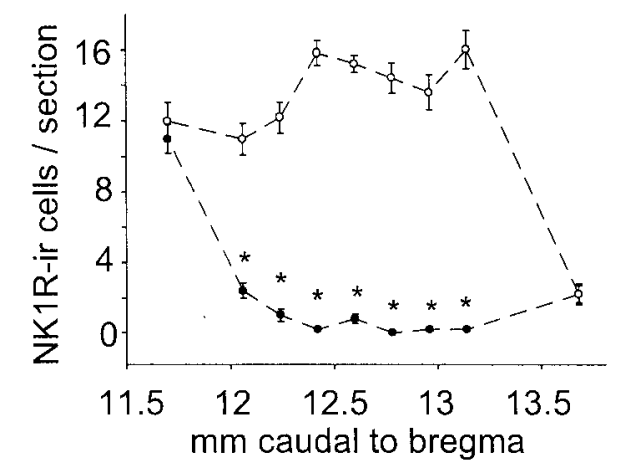

B

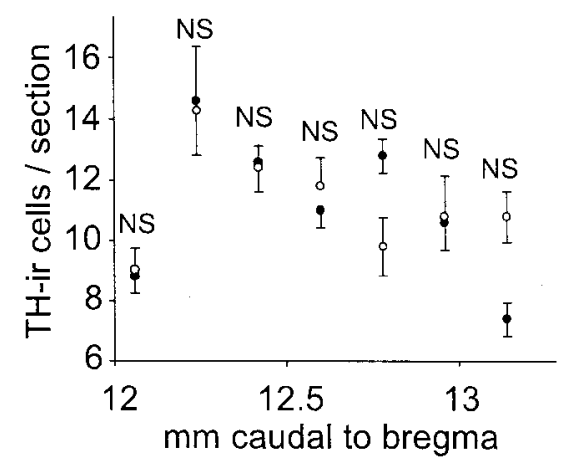

Table 2. Effect of unilateral treatment with SSP-SAP or SAP on the number of TH- or NK1R-ir neurons present within the pre-BötC

\begin{tabular}{lllll} 
& $\begin{array}{l}\text { TH-ir cells } \\
\text { (treated side) }\end{array}$ & $\begin{array}{l}\text { TH-ir cells } \\
\text { (untreated } \\
\text { side) }\end{array}$ & $\begin{array}{l}\text { NK1R-ir } \\
\text { cells (treated } \\
\text { side) }\end{array}$ & $\begin{array}{l}\text { NK1R-ir cells } \\
\text { (untreated side) }\end{array}$ \\
\hline SSP-SAP & $11.9 \pm 0.4$ & $10.8 \pm 0.9$ & $0.4 \pm 0.1^{* \ddagger}$ & $14.8 \pm 0.6$ \\
SAP & $13.7 \pm 0.7$ & $13 \pm 0.7$ & $16.1 \pm 1.2$ & $17.2 \pm 1.1$
\end{tabular}

This table indicates the number of cells found within a $500-\mu \mathrm{m}$-wide $\times 500-\mu \mathrm{m}$-high square box encompassing the pre-BötC in coronal sections. The box was positioned so that the middle of its top side touched the bottom of nucleus amgibuus [for further details, see Wang et al. (2001)]. Two sections (bregma -12.7 and $-12.9 \mathrm{~mm}$ ) were examined per rat, generating one number from which the means were derived. ${ }^{*} p<$ 0.05 when compared with the untreated side of SSP-SAP-treated rats (one-way ANOVA). ${ }^{\ddagger} p<0.05$ when compared with the treated side of SAP-treated rats.

\section{Effects of selective unilateral ablation of the NK1R-ir cells on respiration}

Selective unilateral ablation of the NK1R-ir cells produced little change in basal cardiorespiratory parameters, possibly because, as in many other circuits, destruction of far more than $50 \%$ of a given cell type is required to seriously perturb a circuit (Zigmond and Stricker, 1984; Chiueh et al., 1985). The small increase in basal respiratory rate observed in SSP-SAP-treated rats (Table 1) may be related to the ataxic breathing pattern seen in behaving rats with bilateral lesions of the NK1R-ir cells (Gray et al., 2001).

The respiratory effects produced by DLH injection into the pre-BötC of SSP-SAP-treated rats were clearly abnormal. On the lesioned side, DLH consistently failed to increase respiratory rate, indicating that the integrity of the NK1R-ir cells is essential for the tachypneic response to be elicited. Gray et al. (2001) recently found that rats with bilateral lesions of pre-BötC NK1R-ir cells exhibited a very abnormal breathing pattern when awake (ataxic breathing). These lesioned animals had no spontaneous respiratory drive under anesthesia, but bulbospinal inspira-

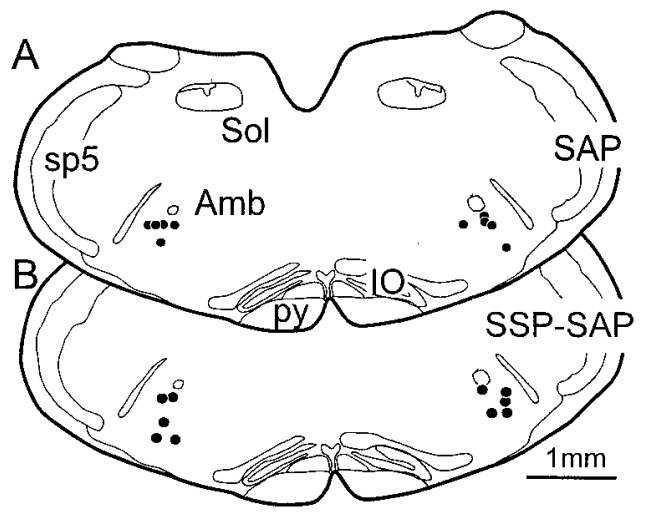

Figure 10. Pre-BötC injection sites in control and experimental rats. These rats received bilateral injections of DLH mixed with fluorescent microbeads into three regions of the VRG (see Figs. 6, 7). This figure only represents the injection sites located at the level of the pre-BötC. $A$, Rats treated unilaterally with SAP. $B$, Rats treated unilaterally with SSP-SAP. See Figure 3 legend for abbreviations.

tory premotor pathways may have been intact. Despite major differences in experimental design, both studies concur that some of the NK1R-ir neurons of the VRG play a critical role in breathing. More specific interpretations of the results of the present study fall into two main categories.

The first possibility is that the NK1R-ir neurons of the preBötC are part and parcel of the respiratory rhythm generating circuitry. Accordingly, some pre-BötC neurons with pre-inspiratory/early inspiratory discharge are NK1R-ir (Guyenet and Wang, 2001). Thus, some of the NK1R-ir cells are synchronized with the respiratory generating circuit, suggesting that they are not merely providing a tonic drive to the oscillator. Because few of the NK1R-ir cells of the pre-BötC are GABAergic or glycin- 
ergic, some of the NK1R-ir neurons of the pre-BötC are likely to be both excitatory and rhythmogenic (Guyenet and Wang, 2001; Wang et al., 2001). Conceivably, some of these cells are the adult equivalent of the glutamatergic rhythm-generating neurons identified in neonate slices (Butera et al., 1999a; Koshiya and Smith, 1999). According to the model of Smith et al. (2000), applying a moderate depolarizing bias to these rhythmogenic neurons increases the single cell and population bursting rate (Del Negro et al., 2001). The tachypnea produced by DLH injection into the pre-BötC therefore could be explained by assuming that this amino acid produces a slight depolarization of this group of excitatory rhythmogenic neurons in vivo.

Alternately, some of the NK1R-expressing neurons of the VRG could be tonic excitatory neurons [as defined by Smith et al. (2000)] that drive the rhythmogenic network. Awake rats with bilateral lesions of VRG NK1R-ir cells experience major difficulty in coping with hypercapnia and hypoxia (Gray et al., 2001). This result could potentially be explained by the loss of neurons that mediate $\mathrm{CO}_{2}$ - or hypoxia-dependent excitatory drive to the respiratory oscillator.

Somewhat unexpectedly, DLH microinjection into the apparently intact side of rats treated unilaterally with SSP-SAP also produced abnormal respiratory responses. This response was characterized by a long period of phrenic apnea that was preceded in some cases by a brief tachypnea and a tonic discharge. One possible interpretation is that local inhibitory neurons become supersensitive to DLH on the unlesioned side because of denervation supersensitivity to glutamate. Accordingly, many NK1R-ir neurons project to the contralateral side, and most of those cells are probably excitatory (Wang et al., 2001). Another possibility is that the phrenic apnea produced by DLH on the intact side is actually caused by excessive depolarization of the underlying excitatory rhythmogenic neurons, and also because the loss of their contralateral input might have rendered glutamatergic receptors supersensitive. Excessive depolarization of these cells produces a tonic discharge that eliminates the population rhythm (Del Negro et al., 2001). Depolarization beyond that point could silence the system because of sodium channel inactivation.

A second unexpected result of the study was that the unilateral lesion of the NK1R-ir cells also changed the effect of DLH injection on arterial pressure. The change was site specific (rVRG and pre-BötC only) and was observed only on the lesioned side. The drop in blood pressure caused by chemical stimulation of the CVLM is attributed to the depolarization of GABAergic propriomedullary neurons that inhibit the presympathetic bulbospinal neurons of the RVLM (Jeske et al., 1993; Dampney 1994). These GABAergic cells are part of the sympathetic baroreflex circuitry, they have a columnar distribution that is coextensive with the pre-BötC and rVRG (between bregma levels -12.3 and $-13.8 \mathrm{~mm}$ ), and they reside just below the VRG (Chan and Sawchenko, 1998). Two main interpretations could account for the reduced hypotension observed when DLH is administered into the rVRG and pre-BötC of rats with unilateral lesion of the NK1R-ir neurons. The simplest one is that some of the aforementioned CVLM GABAergic neurons express NK1 receptors and had been lesioned by SSP-SAP. This possibility is compatible with the existence of a small minority of NK1R-expressing GABAergic neurons in this general region (Wang et al., 2001). Some of these cells clearly reside within the region where NK1R-ir neurons were destroyed. The second interpretation is that some of the NK1R-ir neurons of the ventrolateral medulla provide an excitatory drive to the CVLM GABAergic neurons, which facilitates their depolarization by DLH.

In summary, selective unilateral depletion of the NK1Rexpressing neurons of the VRG alters the cardiorespiratory responses produced by microinjection of DLH in this region. The results suggest that some of the NK1R-ir neurons of the VRG provide an excitatory drive to the respiratory rhythm generator or, perhaps, that they are a component of the rhythm generator. The study also indicates that NK1R-expressing neurons play a role in the neural control of circulation. Further work will be needed to determine whether the same or different NK1Rexpressing neurons control respiration and sympathetic tone.

\section{REFERENCES}

Brown DL, Guyenet PG (1985) Electrophysiological study of cardiovascular neurons in the rostral ventrolateral medulla in rats. Circ Res 56:359-369.

Butera RJJR, Rinzel J, Smith JC (1999a) Models of respiratory rhythm generation in the pre-Botzinger complex. I. Bursting pacemaker neurons. J Neurophysiol 82:382-397.

Butera RJJR, Rinzel J, Smith JC (1999b) Models of respiratory rhythm generation in the pre-Botzinger complex. II. Populations of coupled pacemaker neurons. J Neurophysiol 82:398-415.

Chan RKW, Sawchenko PE (1998) Organization and transmitter specificity of medullary neurons activated by sustained hypertension: implications for understanding baroreceptor reflex circuitry. J Neurosci 18:371-387.

Chitravanshi VC, Sapru HN (1999) Phrenic nerve responses to chemical stimulation of the subregions of ventral medullary respiratory neuronal group in the rat. Brain Res 821:443-460.

Chiueh CC, Burns RS, Markey SP, Jacobowitz DM, Kopin IJ (1985) Primate model of parkinsonism: selective lesion of nigrostriatal neurons by 1-methyl-4-phenyl-1,2,3,6-tetrahydropyridine produces an extrapyramidal syndrome in rhesus monkeys. Life Sci 36:213-218.

Connelly CA, Dobbins EG, Feldman JL (1992) Pre-Botzinger complex in cats: respiratory neuronal discharge patterns. Brain Res 590:337-340.

Dampney RAL (1994) Functional organization of central pathways regulating the cardiovascular system. Physiol Rev 74:323-364.

Del Negro CA, Johnson SM, Butera RJ, Smith JC (2001) Models of respiratory rhythm generation in the pre-Botzinger complex. III. Experimental tests of model predictions. J Neurophysiol 86:59-74.

Dobbins EG, Feldman JL (1994) Brainstem network controlling descending drive to phrenic motoneurons in rat. J Comp Neurol 347:64-86.

Duffin J, Ezure K, Lipski J (1995) Breathing rhythm generation: focus on the rostral ventrolateral medulla. News Physiol Sci 10:133-140.

Ellenberger HH, Feldman JL (1990) Subnuclear organization of the lateral tegmental field of the rat. I: Nucleus ambiguus and ventral respiratory group. J Comp Neurol 294:202-211.

Feldman JL, McCrimmon DR (1999) Neural control of breathing. In: Fundamental neuroscience (Zigmond MJ, Bloom FE, Landis SC, Roberts JL, Squire LR, eds), pp 1063-1090. San Diego: Academic.

Gray PA, Rekling JC, Bocchiaro CM, Feldman JL (1999) Modulation of respiratory frequency by peptidergic input to rhythmogenic neurons in the preBotzinger complex. Science 286:1566-1568.

Gray PA, Janczewski WA, Mellen N, McCrimmon DR, Feldman JL (2001) Normal breathing requires preBotzinger complex neurokinin-1 receptor-expressing neurons. Nat Neurosci 927:927-930.

Guyenet PG, Wang H (2001) Pre-Botzinger neurons with preinspiratory discharges "in vivo" express NK1 receptors in the rat. J Neurophysiol 86:438-446.

Jeske I, Morrison SF, Cravo S, Reis DJ (1993) Identification of baroreceptor reflex interneurons in the caudal ventrolateral medulla. Am J Physiol 264:R169-R178.

Johnson SM, Smith JC, Funk GD, Feldman JL (1994) Pacemaker behavior of respiratory neurons in medullary slices from neonatal rat. J Neurophysiol 72:2598-2608.

Koshiya N, Smith JC (1999) Neuronal pacemaker for breathing visualized in vitro. Nature 400:360-363.

Lieske SP, Thoby-Brisson M, Telgkamp P, Ramirez JM (2000) Reconfiguration of the neural network controlling multiple breathing patterns: eupnea, sighs and gasps. Nat Neurosci 3:600-607.

Liu YY, Ju G, Wong-Riley MT (2001) Distribution and colocalization of neurotransmitters and receptors in the pre-Botzinger complex of rats. J Appl Physiol 91:1387-1395.

Mantyh PW (1997) Inhibition of hyperalgesia by ablation of lamina I spinal neurons expressing the substance $\mathrm{P}$ receptor. Science 278:275-279.

Nakaya Y, Kaneko T, Shigemoto R, Nakanishi S, Mizuno N (1994) 
Immunohistochemical localization of substance $\mathrm{P}$ receptor in the central nervous system of the adult rat. J Comp Neurol 347:249-274.

Paxinos G, Watson C (1998) The rat brain in stereotaxic coordinates, Ed 4. San Diego: Academic.

Pilowsky PM, Feldman JL (2001) Identifying neurons in the preBotzinger complex that generate respiratory rhythm: visualizing the ghost in the machine. J Comp Neurol 434:125-127.

Rekling JC, Shao XM, Feldman JL (2000) Electrical coupling and excitatory synaptic transmission between rhythmogenic respiratory neurons in the preBotzinger complex. J Neurosci 20:RC113(1-5).

Schreihofer AM, Guyenet PG (1997) Identification of C1 presympathetic neurons in rat rostral ventrolateral medulla by juxtacellular labeling in vivo. J Comp Neurol 387:524-536.

Schreihofer AM, Stornetta RL, Guyenet PG (1999) Evidence for glycinergic respiratory neurons: Bötzinger neurons express mRNA for glycinergic transporter 2. J Comp Neurol 407:583-597.

Schreihofer AM, Stornetta RL, Guyenet PG (2000) Regulation of sympathetic tone and arterial pressure by rostral ventrolateral medulla after depletion of Cl cells in rat. J Physiol (Lond) 529:221-236.

Schwarzacher SW, Smith JC, Richter DW (1995) Pre-Bötzinger complex in the cat. J Neurophysiol 73:1452-1461.

Smith JC, Ellenberger HH, Ballanyi K, Richter DW, Feldman JL (1991) Pre-Bötzinger complex: a brainstem region that may generate respiratory rhythm in mammals. Science 254:726-729.
Smith JC, Butera RJ, Koshiya N, Del Negro C, Wilson CG, Johnson SM (2000) Respiratory rhythm generation in neonatal and adult mammals: the hybrid pacemaker-network model. Respir Physiol 122:131-147.

Solomon IC, Edelman NH, Neubauer JA (1999) Patterns of phrenic motor output evoked by chemical stimulation of neurons located in the pre-Bötzinger complex in vivo. J Neurophysiol 81:1150-1161.

Stornetta RL, Guyenet PG (1999) Distribution of glutamic acid decarboxylase mRNA-containing neurons in rat medulla projecting to thoracic spinal cord in relation to monoaminergic brainstem neurons. J Comp Neurol 407:367-380.

Tousignant C, Guillemette G, Drapeau G, Telemaque S, Dion S, Regoli D (1990) 125I-BH[Sar9, Met(O2)11]-SP, a new selective ligand for the NK-1 receptor in the central nervous system. Brain Res 524:263-270.

Wang H, Stornetta RL, Rosin DL, Guyenet PG (2001) Neurokinin-1 receptor-immunoreactive neurons of the ventral respiratory group in the rat. J Comp Neurol 434:128-146.

Wiley RG, Lappi DA (1999) Targeting neurokinin-1 receptorexpressing neurons with [Sar9, Met(O2)11 substance P-saporin. Neurosci Lett 277:1-4.

Willette RN, Punnen-Grandy S, Krieger AJ, Sapru HN (1987) Differential regulation of regional vascular resistance by the rostral and caudal ventrolateral medulla in the rat. J Auton Nerv Syst 18:143-151.

Zigmond MJ, Stricker EM (1984) Parkinson's disease: studies with an animal model. Life Sci 35:5-18. 